\title{
Hydrophobic nano-carrier for lysozyme adsorption
}

\author{
CANAN ALTUNBAŞ ${ }^{1}$, FULDEN ZEYNEP URAL ${ }^{1}$, MURAT UYGUN ${ }^{2}$, NESİBE AVCIBAŞI ${ }^{3}$, \\ UĞUR AVCIBAŞI ${ }^{4}$, DENIZ AKTAŞ UYGUN ${ }^{5, *}$ and SINAN AKGÖL ${ }^{1}$ \\ ${ }^{1}$ Department of Biochemistry, Ege University, 35040 İzmir, Turkey \\ ${ }^{2}$ Kocarlı Vocational and Training School, Adnan Menderes University, 09010 Aydın, Turkey \\ ${ }^{3}$ Ege Higher Vocational School, Ege University, 35040 İzmir, Turkey \\ ${ }^{4}$ Department of Chemistry, Faculty of Arts and Science, Celal Bayar University, 45140 Manisa, Turkey \\ ${ }^{5}$ Department of Chemistry, Faculty of Arts and Science, Adnan Menderes University, 09010 Aydın, Turkey
}

MS received 5 October 2015; accepted 29 October 2015

\begin{abstract}
In this work, poly(HEMA-APH) nanoparticles were synthesized by surfactant-free emulsion polymerization technique. Magnetic behaviour was introduced by simple addition of $\mathrm{Fe}_{3} \mathrm{O}_{4}$ into the polymerization medium. Characterization of the nanoparticle was carried out by FTIR, ESR, SEM, AFM and EDX analyses. These synthesized magnetic nanoparticles were used for adsorption of lysozyme. For this purpose, adsorption conditions were optimized and maximum lysozyme binding capacity was found to be $278.8 \mathrm{mg} \mathrm{g}^{-1}$ polymer in $\mathrm{pH} 7.0 \mathrm{phosphate}$ buffer at $25^{\circ} \mathrm{C}$. Desorption and reusability properties of the nanoparticles were investigated and lysozyme adsorption efficiency did not change significantly at the end of the $\mathbf{1 0}$ successive reuses.
\end{abstract}

Keywords. Nanoparticle; lysozyme; adsorption; poly(HEMA-APH).

\section{Introduction}

Lysozyme (EC 3.2.1.17) is a self-defense enzyme for protection from microorganisms and is produced in many vertebrates' organs and serum and mucus. However, lysozyme is a very small enzyme $(14.4 \mathrm{kDa})$ and its industrial applications are very valuable. For e.g., they have been used as a celldisrupting agent, antibacterial agent and food additives [1-3]. Recently, lysozyme has been used as an anti-cancer agent $[4,5]$. Because of its precious properties, purification and separation of lysozyme have been taken great interest and high efficient, simple and cheap techniques have been developed to get the highly purified lysozyme [6-9].

Hydrophobic interaction chromatography (HIC) is a simple and specific method used for the separations of proteins in laboratory scale [10-13]. It was first described by Shepard and Tiselius in 1949 [14] and defined as 'saltingout chromatography' as they used salt solutions for elutions. Later, Shaltiel and Er-el $[15,16]$ renamed this technique as 'hydrophobic chromatography' and Hofstee [17] called this as 'hydrophobic adsorption chromatography'. Finally, Hjerten et al [18] made last point and presented the concept as 'hydrophobic interaction chromatography' to describe the separation of proteins adsorbed onto hydrophobic medium by salts [19]. HIC is based on the interaction between hydrophobic ligand carrying support material and hydrophobic amino acids bearing non-polar regions of protein surface [20]. The main driving force for this interaction is van der Waals interactions [21]. Solvophobic theory $[22,23]$ and preferential interaction

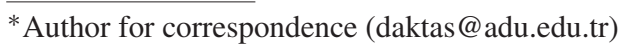

theory $[24,25]$ have been used for describing the separation mechanism.

Recently, synthesis and production of nanosized materials in various shapes and sizes have been taken great interest. They have been intensively used in diverse biotechnological applications such as in sensor and purification techniques due to their unique physical and chemical properties. As wellestablished nanomaterials and magnetic nanoparticles tender advantageous properties such as controlled size, easy mobility and direction with external magnetic field, and improved contrast in magnetic resonance imaging. Because of its unique properties and easy preparation features, magnetic nanoparticles have been chosen as support materials in various biotechnological applications such as detection of bacteria, purification of biomolecules, immobilization of enzymes, decorporation of contaminants, drug delivery, cancer treatment with hyperthermia, etc. [26]. Magnetic nanoparticles have been used for enzyme adsorption applications due to their advantageous features, for e.g., they serve very high surface area and so their protein adsorption capacities are very high; their mass transfer resistance are relatively low and their pollution yields are very low; they can easily be removed from the adsorption medium by simple external magnetic field and this serves as selective separation of immobilized enzymes [26-31].

There are great varieties of chemical and physical methods for immobilization of an enzyme onto solid carrier. Within these immobilization methods for enzyme, adsorption is the most important and useful one. Driving forces for adsorption process are physical interactions between carrier and enzyme molecule such as van der Waals, ionic interactions, 
hydrophobic interactions and hydrogen bonding [32-34]. Hydrophobic interaction has been used as an alternative for lysozyme adsorption and this paper reports the adsorption of lysozyme by using hydrophobic ligand attached magnetic nanoparticles. For this, poly(HEMA-APH) magnetic nanoparticles were synthesized by surfactant-free emulsion polymerization technique by using 2-hydroxylethyl methacrylate (HEMA) as a structural monomer, anilinephtalein (APH) as a functionalization monomer and $\mathrm{Fe}_{3} \mathrm{O}_{4}$ as a magnetic component. Characterization of the synthesized magnetic nanoparticles was carried out by Fouirer transform infrared spectroscopy (FTIR), electron spin resonance (ESR), scanning electron microscope (SEM), atomic force microscope (AFM), zeta size analysis and energy dispersive X-ray (EDX) analyses. Synthesized magnetic nanoparticles were used for the adsorption of lysozyme from its aqueous solutions and medium conditions were changed to reach the maximum lysozyme adsorption yield. For this; $\mathrm{pH}$ of medium, initial lysozyme concentration, temperature and ionic strength are varied and their effects on the lysozyme adsorption capacity are studied. Also, desorption and reusability profile of magnetic poly(HEMA-APH) nanoparticles were investigated.

\section{Materials and methods}

\subsection{Materials}

Lysozyme (from chicken egg white, EC 3.2.1.17), Micrococcus lysodeikticus (lyophilized), nitrobenzene, phthaloyl chloride, $\mathrm{Fe}_{3} \mathrm{O}_{4}$ (average diameter, 20-50 nm) were supplied from Sigma (St. Louis, USA). 2-Hydroxyethyl methacrylate (HEMA) and ethylene glycol dimethacrylate (EGDMA) were purchased from Fluka (Switzerland). All other chemicals were of analytical grade and obtained from Merck (Darmstad, Germany). Deionized ultrapure water was used for all solutions (Millipore, Simplicity, $18.2 \mathrm{~m} \Omega \mathrm{cm}^{-1}$ ).

\subsection{Methods}

2.2a Preparation of APH monomer: Synthesis of functional monomer APH was carried out by the procedure of condensation reaction by Hubacher [35] with minor modifications [36]. Briefly, $10 \mathrm{~g}$ of $\mathrm{AlCl}_{3}$ was mixed with $50 \mathrm{ml}$ of nitrobenzene. Then, $4.06 \mathrm{~g}$ of phthalyl chloride and $4.24 \mathrm{~g}$ of s-diphenylurea were added to the initial mixture and heated to $80^{\circ} \mathrm{C}$ for $30 \mathrm{~min}$. Nitrobenzene phase was removed by steam distillation and condensation product was obtained. Then this product was refluxed with acetic acid, water and $\mathrm{H}_{2} \mathrm{SO}_{4}$ for $4 \mathrm{~h}$. Residual product was then dissolved in boiling $\mathrm{HCl}(1.0 \mathrm{~N})$ and mixed with water and cooled down to $5^{\circ} \mathrm{C}$. Filtrated material was washed with $\mathrm{NH}_{4} \mathrm{OH}$ and crystallized by methanol. Further characterization of the APH monomer can be found in the literature of our research group [36].

2.2b Preparation of magnetic poly(HEMA-APH) nanoparticles: Magnetic poly(HEMA-APH) nanoparticles were synthesized by surfactant-free polymerization method [36,37]. Briefly, $0.5 \mathrm{~g}$ of PVA dissolved in $45 \mathrm{ml}$ of water and transferred to a polymerization reactor. Then, polymerized solution was prepared by the addition of $4.96 \mathrm{mmol}$ of HEMA, $1.59 \mathrm{mmol}$ of EGDMA, $0.25 \mathrm{mmol}$ of APH and $100 \mathrm{mg}$ of $\mathrm{Fe}_{3} \mathrm{O}_{4}$. Potassium peroxodisulfate was used as an initiator and was added to the polymerized solution $(45 \mathrm{ml}$, $0.0198 \mathrm{~g}$ ). Polymerization was carried out in a shaking water bath at $70^{\circ} \mathrm{C}$ for $24 \mathrm{~h}$ with the shaking rate of $65 \mathrm{rpm}$. At the end of the polymerization reaction, polymers were washed with ethanol and water to remove the unreacted monomers and initiators. Preparation of the magnetic poly(HEMA-APH) nanoparticles is schematically demonstrated in figure 1.

\section{2c Characterization of magnetic-poly(HEMA-APH)} nanoparticles: Characteristic IR region bands of the synthesized nanoparticles were obtained by using a FTIR spectrophotometer (Spectrum 100, Perkin-Elmer, USA). For this, first nanoparticles were dried at oven, grounded and were mixed with IR-grade $\mathrm{KBr}$. Then, the mixture was pressed into a pellet form and mounted in the FTIR spectrophotometer and spectrum was recorded. ESR was used to show the presence of magnetic behaviour by using ESR devices (Varian, EL9, USA). Size and the size distribution of the nanoparticles were examined by Nano Zetasizer (Malvern Instrument, NanoS, England). Surface characteristics were also investigated by SEM studies. For SEM,
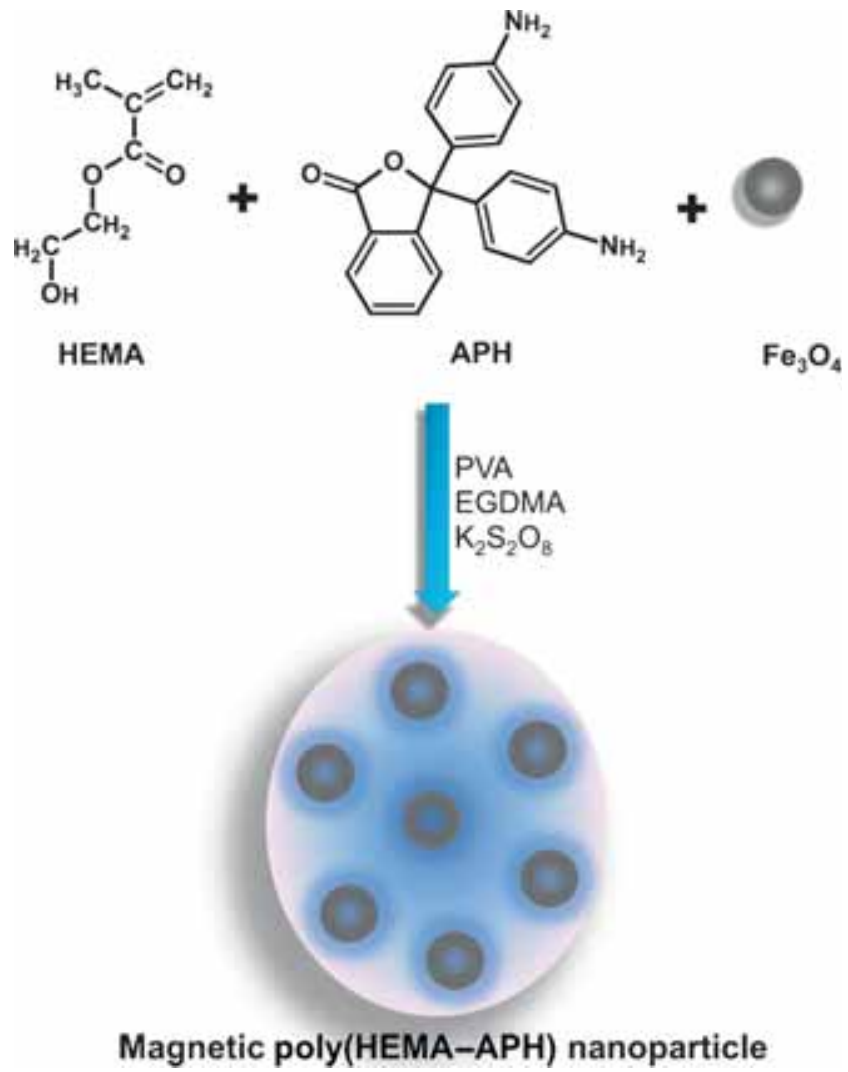

Figure 1. Schematic presentation of synthesis of magnetic poly (HEMA-APH) nanoparticles. 
nanoparticles were dried, covered with thin layer of gold and pictures were taken by SEM devices (Philips, XL-30S FEG, The Netherlands). Surface morphology and shape of the synthesized nanoparticles were determined by AFM. For this, the nanoparticles were precipitated and collected onto a glass plate $(1 \times 1 \mathrm{~cm})$ and then dried. AFM pictures of the nanoparticles were taken by using AFM devices (Digital Instruments, MMSPM, Nanoscope VI, USA) with tapping mode. Incorporation of $\mathrm{APH}$ monomer and $\mathrm{Fe}_{3} \mathrm{O}_{4}$ onto polymeric structure was realized by using EDX analysis. For this, dried samples were mounted into EDX instrument (Philips XL 30 S FEG) and chosen area was scanned for analysis.

2.2d Lysozyme adsorption-desorption studies from aqueous solutions: Lysozyme adsorption studies were carried out by using batch-wise set up. All adsorption experiments were performed at $25^{\circ} \mathrm{C}$ for $2 \mathrm{~h}$ at the stirring rate of $120 \mathrm{rpm}$. To optimize the maximum bounded amount of lysozyme, some system parameters were changed such as $\mathrm{pH}(5-10)$, lysozyme concentration $\left(0.1-1.5 \mathrm{mg} \mathrm{ml}^{-1}\right)$, temperature (4$\left.45^{\circ} \mathrm{C}\right)$ and ionic strength $(0-1 \mathrm{M})$. Lysozyme concentrations at initial and final adsorption medium were measured spectrophotometrically at $280 \mathrm{~nm}$ by using a double beam UV-Vis spectrophotometer (Shimadzu, UV-1601, Japan). Adsorbed amount of lysozyme onto nanoparticles was determined by subtraction of final lysozyme concentration from the initial concentration. Desorption of lysozyme from the nanoparticles were also studied and ethylene glycol $(50 \%)$ was used as a desorption agent. Reusability of the magnetic nanoparticles was demonstrated by ten successive adsorption-desorption cycles by using same nanoparticles.

2.2e Assay of lysozyme activity: A simple spectrophotometric method from Shugar [38] was used for the activity tests. For this, Micrococcus lysodeikticus suspension was prepared by mixing $9 \mathrm{~g}$ of $M$. lysodeikticus with $30 \mathrm{ml}$ of

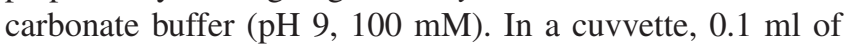
lysozyme solution was mixed with $2.9 \mathrm{ml}$ of cell suspension and cell lysis was monitored spectrophotometrically at $450 \mathrm{~nm}$ for $5 \mathrm{~min}$. One lysozyme activity unit was defined as the decrease in absorbance for 0.001 units at $450 \mathrm{~nm}$ (by using $\mathrm{pH} 9$ carbonate buffer at $25^{\circ} \mathrm{C}$ ).

\section{Results and discussion}

Support materials play very important role for enzyme immobilization studies. Magnetic nano-sized support materials have taken great interest because of their unique properties such as very high specific surface area, low mass transfer resistance, reusability properties with an external magnetic field and easy modification with various reactive groups, etc. [39-41]. Figure 2 shows FTIR-ATR spectrum of the

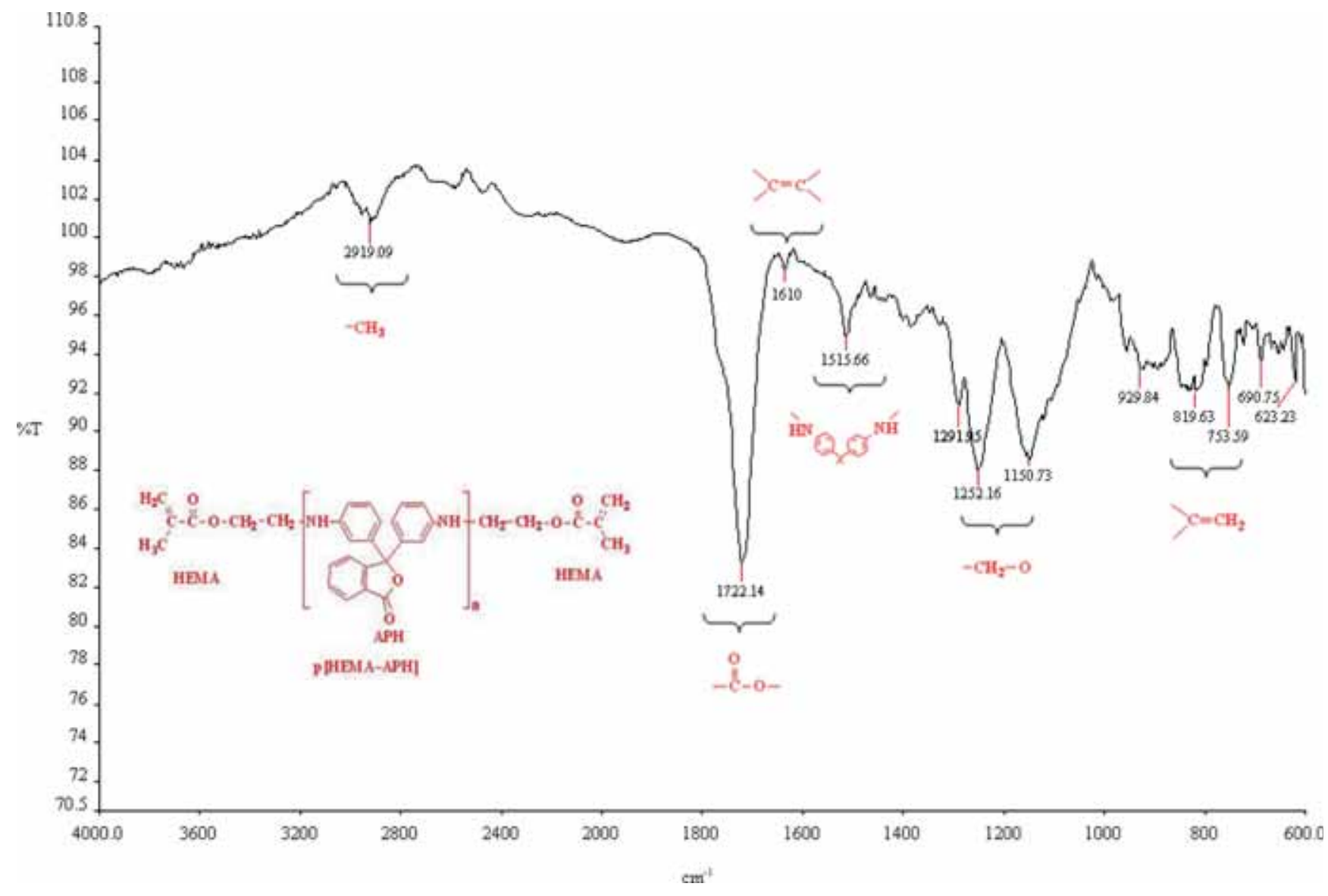

Figure 2. FTIR spectrum of poly(HEMA-APH) nanoparticles. 


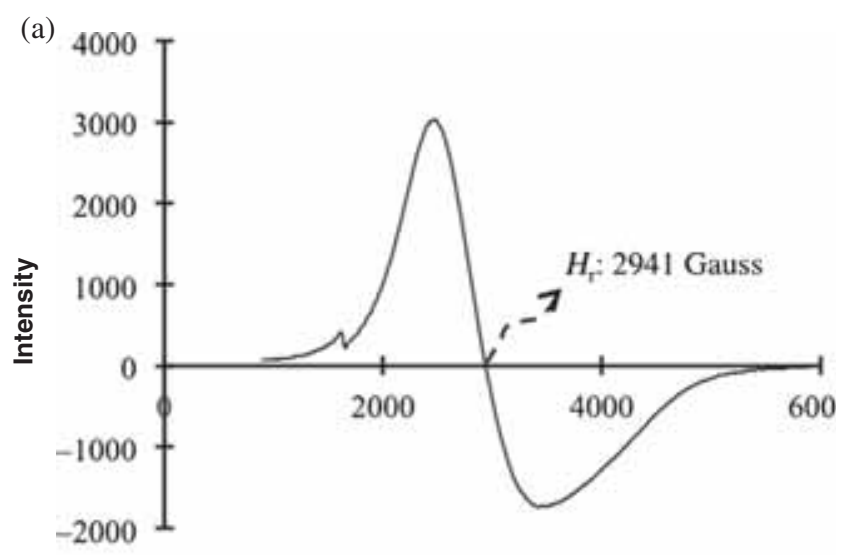

Magnetic field, Gauss

(b)

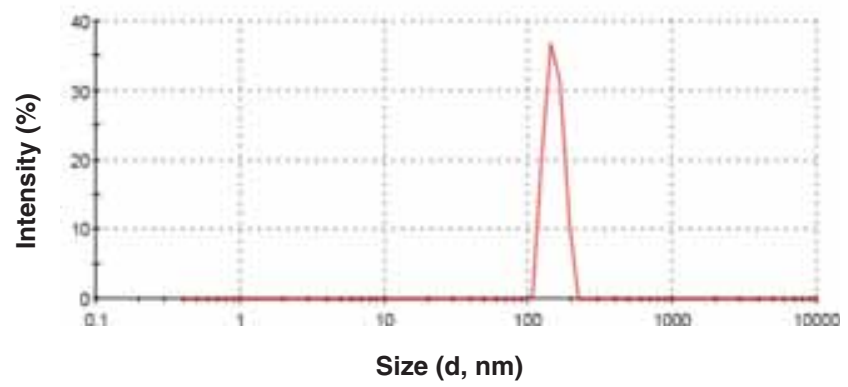

Figure 3. ESR spectrum (a) and zeta size analysis (b) of poly(HEMA-APH) nanoparticles.

synthesized poly(HEMA-APH) nanoparticles. As seen here, stretching bands of free- $\mathrm{CH}_{3}$ groups were located at around $2900 \mathrm{~cm}^{-1}$, while stretching bands of ester groups were located at around $1700 \mathrm{~cm}^{-1}$. Bending bands of $-\mathrm{NH}$ group of aromatic structure related to amine groups were seen at $1515 \mathrm{~cm}^{-1}$. This finding also contributed to the binding of amine group bearing APH monomer to the HEMA backbone via $-\mathrm{OH}$ groups. Magnetic properties of the poly(HEMAAPH) nanoparticles were demonstrated by using ESR analysis. Figure 3a shows the ESR spectrum of poly(HEMAAPH) nanoparticles. By using this spectrum, $g$ factor of the nanoparticles was calculated as 2.359 . This value means that the synthesized nanoparticles had a local magnetic field because of the magnetite particles attached in the polymer structure. Also, it can be concluded from this results that these magnetic nanoparticles could be easily directed by external magnetic field. Size distribution and average particle size were investigated by using Zeta Sizer and particle size was calculated as $150 \mathrm{~nm}$ (figure 3b). Shape, size and surface morphology of the synthesized magnetic nanoparticles were investigated by using SEM and AFM analyses. SEM picture of the nanoparticle is demonstrated in figure 4a. As seen here, synthesized nanoparticles were spherical in shape and their average diameter was found to be around 100-150 nm. Figure $4 \mathrm{~b}$ shows the AFM picture of the magnetic poly(HEMA-APH) nanoparticles. As clearly seen here, nanoparticles were spherical in shape and they were in nanometre
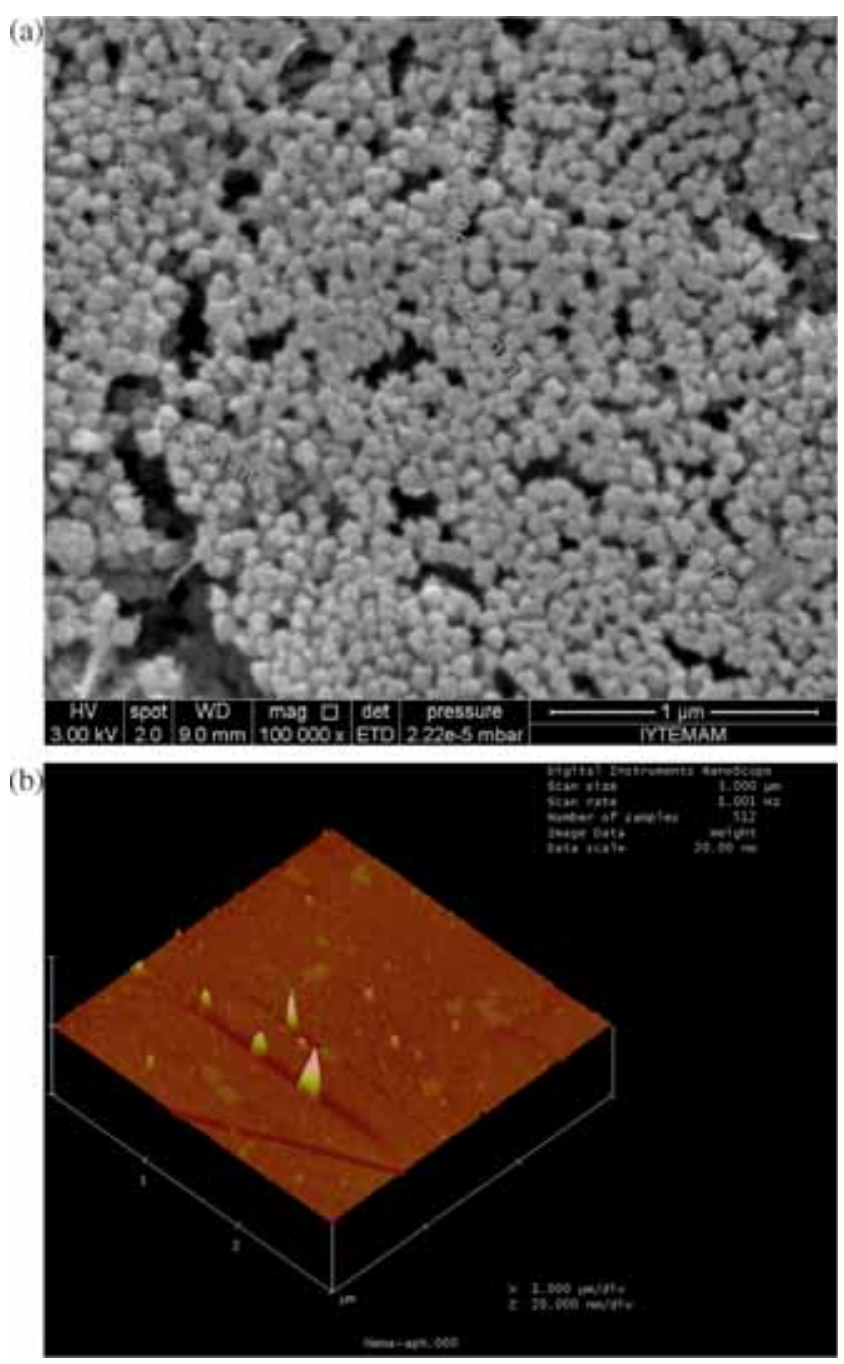

Figure 4. (a) SEM and (b) AFM photographs of poly(HEMAAPH) nanoparticles.

size. EDX analysis was used for the assessment of the incorporation of APS and $\mathrm{Fe}_{3} \mathrm{O}_{4}$ in the nanoparticle structure (figure 5). As seen in this figure, synthesized nanoparticles consisted of $\mathrm{C}, \mathrm{O}, \mathrm{N}$ and $\mathrm{Fe}$ atoms. As clearly seen here, $\mathrm{Fe}_{3} \mathrm{O}_{4}$ particles were successfully attached to the polymeric structure. Because $\mathrm{N}$ atoms only come from APS monomers, it can be concluded that APS monomer was successfully incorporated to the polymeric backbone.

\subsection{Adsorption of lysozyme from aqueous solutions}

Effect of medium $\mathrm{pH}$ on the adsorption of lysozyme onto magnetic poly(HAME-APH) nanoparticles is shown in figure 6. As shown here, maximum lysozyme adsorption capacity was found to be $270 \mathrm{mg} \mathrm{g}^{-1}$ polymer by using $\mathrm{pH} 7$ phosphate buffer. There are one histidine, four aspartic acid, two glutamic acid and two tyrosine amino acid residues on the accessible surface of the lysozyme molecule. However, the driving force for binding was hydrophobic, these amino acid residues could also be contributed to the interaction between 


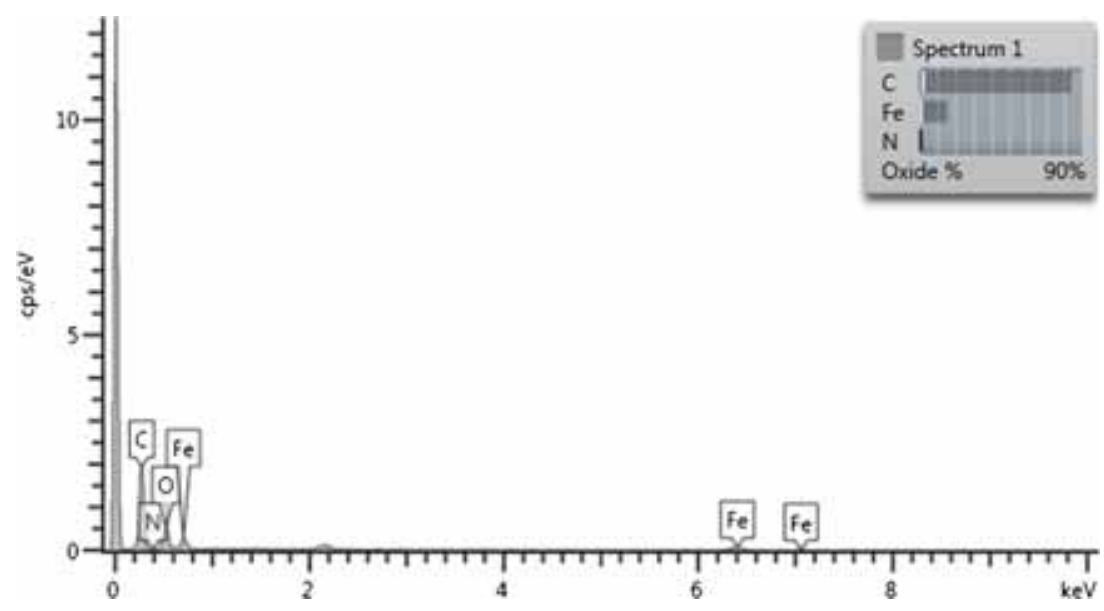

Figure 5. EDX spectrum of poly(HEMA-APH) nanoparticles.

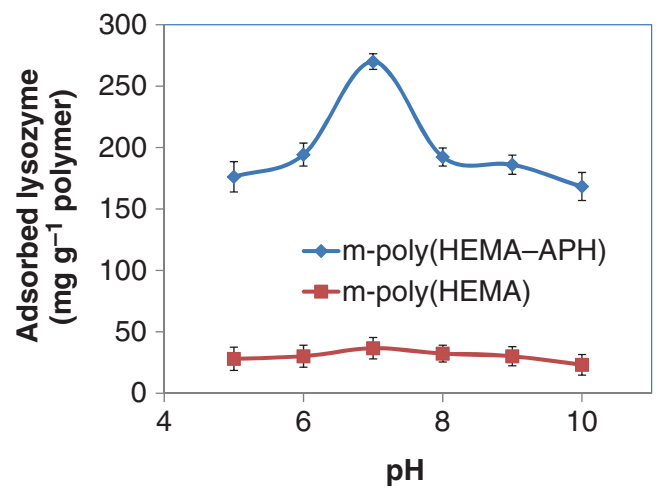

Figure 6. Effect of medium $\mathrm{pH}$ on the lysozyme adsorption onto magnetic poly(HEMA-APH) nanoparticles. Initial lysozyme concentration: $0.5 \mathrm{mg} \mathrm{ml}^{-1}$; temperature: $25^{\circ} \mathrm{C}$; incubation time: $2 \mathrm{~h}$.

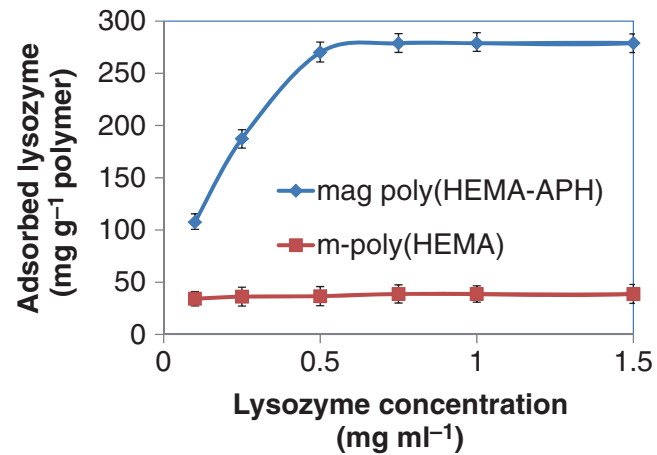

Figure 7. Effect of initial lysozyme concentration on the lysozyme adsorption onto magnetic poly(HEMA-APH) nanoparticles. $\mathrm{pH}$ : 7 ; temperature: $25^{\circ} \mathrm{C}$; incubation time: $2 \mathrm{~h}$.

the lysozyme molecule and the magnetic nanoparticle [42]. The main hydrophobic constituent of the nanoparticle was APH monomer. Plain magnetic poly(HEMA) nanoparticles were also synthesized and lysozyme adsorption capacity of this un-modified nanoparticle was found to be negligible (36.68 $\mathrm{mg} \mathrm{g}^{-1}$ polymer at $\mathrm{pH} 7$ ).
Effect of initial lysozyme concentration onto lysozyme adsorption onto the nanoparticles also demonstrated in figure 7. As shown here, adsorbed amount of lysozyme increased almost linearly with the increasing lysozyme concentration up to $0.5 \mathrm{mg} \mathrm{ml}^{-1}$, then adsorbed amount reached a saturation value. This plateau value taken place because of the fact that all the binding regions of the nanoparticle were occupied with lysozyme molecules and could not bind any more lysozyme. The highly sloped initial part of the adsorption isotherm represents the high affinity between the lysozyme and APH-modified magnetic nanoparticle. As seen here, maximum lysozyme adsorption value was calculated as $278.8 \mathrm{mg} \mathrm{g}^{-1}$ polymer. Also seen in this figure is that non-specific adsorption of lysozyme onto plain poly(HEMA) nanoparticle was very low $\left(38.8 \mathrm{mg} \mathrm{g}^{-1}\right.$ polymer).

Adsorption isotherms are used for the evaluation of the adsorption process. For this, results of the lysozyme adsorption experiment were converted to two main adsorption isotherm equations; Langmuir and Freundlich and expressed as following equations, respectively:

$$
\begin{aligned}
& \text { Langmuir isotherm: } \frac{1}{q_{\mathrm{e}}}=\left(\frac{1}{Q_{\max }}\right)+\left(\frac{1}{Q_{\max } b}\right)\left(\frac{1}{C_{\mathrm{e}}}\right), \\
& \text { Freundlich isotherm: } \ln q_{\mathrm{e}}=\frac{1}{n\left(\ln C_{\mathrm{e}}\right)}+\ln K_{\mathrm{F}},
\end{aligned}
$$

here $b$ and $K_{\mathrm{F}}$ are constants for Langmuir and Freundlich isotherms, respectively, $n$ is Freundlich exponent which expresses the heterogeneity of surface, $q_{\mathrm{e}}$ the adsorbed amount of lysozyme and $q_{\max }$ the maximum loading capacity of the adsorption matrix. Kinetic constants of isotherms are summarized in table 1 . As seen here, it was easily concluded by comparing the $R^{2}$ values of the isotherms and adsorption process was accordant with Langmuir isotherm. In the light of this finding, it can be expressed that adsorption of lysozyme on the magnetic nanoparticle was homogeneous. Lysozyme binding sites had equal affinity and there was no interaction between the adsorbed lysozyme molecules. 
Table 1. Kinetic constants for Langmuir and Freundlich isotherms.

\begin{tabular}{lccccccc}
\hline \multicolumn{3}{c}{ Langmuir } & & \multicolumn{4}{c}{ Freundlich } \\
\cline { 1 - 2 } \cline { 5 - 7 }$q_{\max }\left(\mathrm{mg} \mathrm{g}^{-1}\right)$ & $b\left(\mathrm{ml} \mathrm{mg}^{-1}\right)$ & $R^{2}$ & & $K_{\mathrm{F}}\left(\mathrm{mg} \mathrm{g}^{-1}\right)$ & $1 / n$ & $R^{2}$ \\
\hline 308.64 & 0.1167 & 0.9989 & & 1.044 & 0.9475 & 0.9499 \\
\hline
\end{tabular}

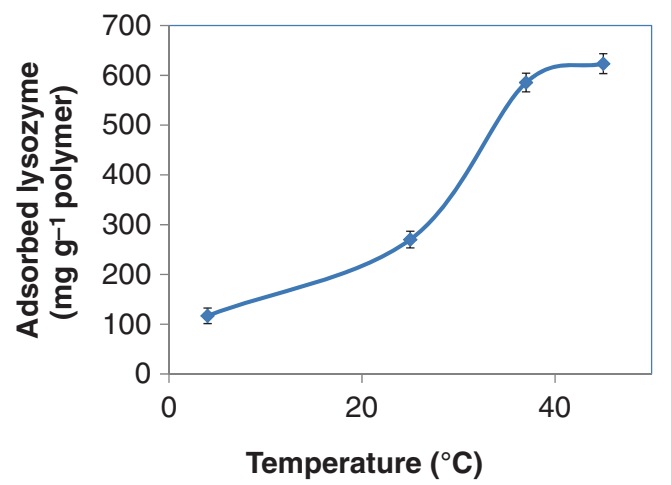

Figure 8. Effect of medium temperature on the lysozyme adsorption magnetic poly(HEMA-APH) nanoparticles. Initial lysozyme concentration: $0.5 \mathrm{mg} \mathrm{ml}^{-1}$; $\mathrm{pH}$ : 7; incubation time: $2 \mathrm{~h}$.

Figure 8 represents the effect of temperature on the adsorbed amount of lysozyme onto magnetic poly(HEMAAPH) nanoparticles. As clearly seen in this figure that adsorbed amount of lysozyme increased from 116.88 to 623.42 with increasing medium temperature from 4 to $45^{\circ} \mathrm{C}$. If hydrophobic interactions are dominant, bounded amount of adsorbent increases with increased temperature. Hjerten et al [18] reported in their works about 'hydrophobic interaction chromatography' (HIC) that high temperature promotes protein retention and low temperature enhances protein elution. It can be clearly concluded from this result that the main driving force for the lysozyme adsorption was hydrophobic interactions.

In hydrophobic interaction studies, one of the most important parameters is ionic strength of the adsorption solution. In this study, $\mathrm{NaCl}$ was used as a salt and figure 9 demonstrates the effect of salt concentration on the lysozyme adsorption. As seen here, adsorbed amount of lysozyme increased with increased concentrations of $\mathrm{NaCl}$ and maximum lysozyme adsorption was found to be $497.93 \mathrm{mg} \mathrm{g}^{-1}$ polymer by using $1 \mathrm{M}$ of $\mathrm{NaCl}$.

\subsection{Reusability and stability of the poly(HEMA-APH) magnetic nanoparticles}

Because of the economic reasons, it is very important to prepare low cost, reusable and highly effective adsorbents. To desorb the adsorbed lysozyme from the magnetic nanoparticle, a solution of ethylene glycol (50\%) was used in a batch mode. By this way, $86 \%$ of adsorbed lysozyme could be

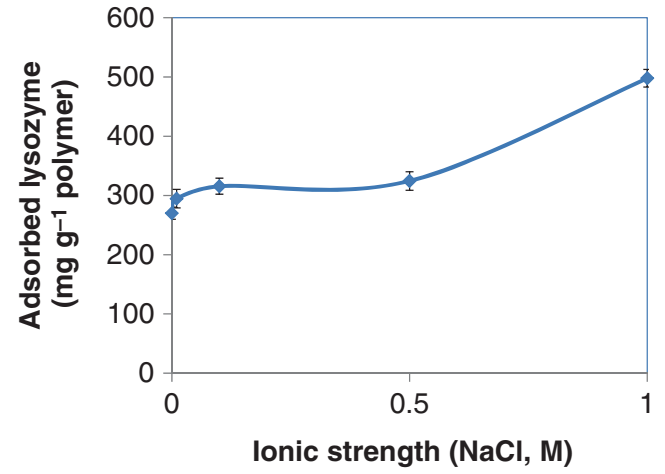

Figure 9. Effect of medium ionic strength on the lysozyme adsorption onto magnetic poly(HEMA-APH) nanoparticles. Initial lysozyme concentration: $0.5 \mathrm{mg} \mathrm{ml}^{-1}$; $\mathrm{pH}$ : 7 ; temperature: $25^{\circ} \mathrm{C}$; incubation time: $2 \mathrm{~h}$.

desorbed. Desorbed nanoparticles were washed two times with distilled water and equilibrated with $\mathrm{pH} 7$ phosphate buffer. These rinsed nanoparticles could be used for the next adsorption steps. To examine the reusability, adsorption/desorption cycle was repeated for ten times. It was founded by the reusability studies that lysozyme adsorption capacity of the magnetic nanoparticles was not significantly changed, at the end of ten reuses. This finding is also demonstrates that these newly synthesized nanoparticles can be used repeatedly without any decrease in their lysozyme adsorption capacities.

\section{Conclusion}

Nanosized particles have been intensively used in various biotechnological applications due to their unique properties and high surface areas. Nanoparticles with magnetic properties can be easily separated from the adsorption mixture by external magnetic field. By this way, analyte molecule can be adsorbed and separated with one single step. One of the efficient adsorption technique used for diverse biotechnological applications is hydrophobic interaction and in this present work, hydrophobic nanoparticles were synthesized by hydrophobic monomer APH. These hydrophobic supports were used for capturing and separation of lysozyme. Lysozyme is a very valuable enzyme and has various important biotechnological applications. In this work, magnetic poly(HEMA-APH) nanoparticles were synthesized, characterized and used for lysozyme adsorption studies. Optimum lysozyme adsorption conditions were determined and maximum lysozyme adsorption efficiency of the magnetic nanoparticle was $278.8 \mathrm{mg} \mathrm{g}^{-1}$ polymer at $25^{\circ} \mathrm{C}$ in $\mathrm{pH} 7$ phosphate buffer. Reusability property of the nanoparticle was also determined and adsorption capacity did not change significantly at the end of the 10 successive reuses. By these results from this study, it can be concluded that these novel magnetic nanoparticles can find appropriate place in hydrophobic separation of biomolecules in biotechnological studies. 


\section{References}

[1] Jing T, Du H, Dai Q, Xia H, Niu J, Hao Q, Mei S and Zhou Y 2010 Biosens. Bioelectron. 26301

[2] Freitas D S and Abrahano-Neto J 2010 Int. J. Pharm. 392111

[3] Zhang G, Cao Q, Li N, Li K and Liu F 2011 Talanta 83 1515

[4] Murakami F, Sasaki T and Sugahara T 1997 Cytotechnology 24177

[5] Uygun D A, Karagözler A A, Akgöl S and Denizli A 2009 Mat. Sci. Eng. C 292165

[6] Cömert Ş C and Odabaşı M 2014 Mat. Sci. Eng. C 341

[7] Altıntaş E B and Denizli A 2006 Int. J. Biol. Macromol. 3899

[8] Odabaş1 M and Denizli A 2004 J. Appl. Polym. Sci. 93719

[9] Odabaşı M, Say R and Denizli A 2007 Mat. Sci. Eng. C 2790

[10] Hearn M T W 2002 in HPLC of biological macromolecules (eds) K M Gooding and F E Regnier (New York: Marcel Dekker) Chapter 5

[11] Jungbauer A and Feng W 2002 in: HPLC of biological macromolecules (eds) K M Gooding and F E Regnier (New York: Marcel Dekker)

[12] Queiroz J A, Tomaz C T and Cabral J M S 2005 J. Biotechnol. 87143

[13] Jungbauer A, Machold C and Hahn R 2005 J. Chromatogr. A 1079221

[14] Shepard C C and Tiselius A 1949 Discussions of the Faraday Society, Vol. 7 (London: Hazell Watson and Winey) p 275

[15] Er-el Z and Shaltiel S 1974 FEBS Lett. 40142

[16] Shaltiel S and Er-el Z 1973 Proc. Natl. Acad. Sci. 70778

[17] Hofstee B H J 1973 Anal. Biochem. 52430

[18] Hjerten S, Rosengren J and Pahlman S J 1974 J. Chromatogr. A 101281

[19] Mahn A, Lienqueo M E and Salgado J C 2009 J. Chromatogr. A 12161838

[20] Queiroz J A, Tomaz C T and Cabral J M S 2001 J. Biotechnol. 87143
[21] Van Oss C J, Good R J and Chaudhury M K 1986 J. Colloid Interface Sci. 111378

[22] Melander W R, Conrradi D and Horvath C 1984 J. Chromatogr. 31767

[23] Diogo M M, Queiroz J A and Prazeres D M F $2002 \mathrm{~J}$. Chromatogr. A 944119

[24] Arakawa T and Timasheff S N 1982 Biochemistry 216545

[25] Freitas S S, Santos J A L and Prazeres D M F 2009 Sep. Purif. Technol. 6595

[26] Huang S-H and Juang R-S 2011 J. Nanopart. Res. 13 4411

[27] Namdeo M and Bajpai S K 2009 J. Mol. Catal. B 59 134

[28] Sarı M, Akgöl S, Karataş M and Denizli A 2006 Ind. Eng. Chem. Res. 453036

[29] Akgöl S, Bereli N and Denizli A 2005 Macromol. Biosci. 5 786

[30] Akgöl S, Türkmen D and Denizli A 2004 J. Appl. Polym. Sci. 932669

[31] Halling P J and Dunnill P 1980 Enzyme Microb. Technol. 22

[32] Cao L 2005 Curr. Opin. Biotechnol. 9217

[33] Sheldon R A 2007 Adv. Synth. Catal. 491289

[34] Jesionowski T, Zdarta J and Krajewska B 2014 Adsorption 20 801

[35] Hubacher M H 1951 J. Am. Chem. Soc. 735885

[36] Avcıbaşı U, Avcıbaşı N, Akalın H A, Ediz M, Demiroğlu H, Gümüşer F G et al 2013 J. Nanopart. Res. 152021

[37] Uygun M, Uygun D A, Altunbaş C, Akgöl S and Denizli A 2014 Sep. Sci. Technol. 491270

[38] Shugar D 1952 Biochim. Biophys. Acta 8302

[39] Xie W and Ma N 2009 Energy Fuel 231347

[40] Liu T, Zhao Y, Wang X, Li X and Yan Y 2013 Bioresour. Technol. 13299

[41] Cui R, Bai C, Jiang Y, Hu M, Li S and Shai Q 2015 Chem. Eng. J. 259640

[42] Yilmaz F, Bereli N, Yavuz H and Denizli A 2009 Biochem. Eng. J. 43272 